\title{
HPLC method development for fampridine using Analytical Quality by Design approach
}

BÉLA KOVÁCS ${ }^{1,2}$

FRANCISC BODA ${ }^{3}$

IBOLYA FÜLÖP ${ }^{4, *}$

ISTVÁN SZÉKELY-SZENTMIKLÓSI ${ }^{1,5}$

ÉVA KATALIN KELEMEN ${ }^{1}$

BOGLÁRKA KOVÁCS-DEÁK²

BLANKA SZÉKELY-SZENTMIKLÓSI ${ }^{\wp}$

${ }^{1}$ Gedeon Richter Romania, 540306

Tîrgu Mureș, Romania

${ }^{2}$ Department of Biochemistry and the Chemistry of Environmental Factors, Faculty of Pharmacy

University of Medicine, Pharmacy, Science and

Technology of Tîrgu Mureș

540139, Tîrgu Mureș, Romania

${ }^{3}$ Department of General and Inorganic Chemistry, Faculty of Pharmacy, University of Medicine, Pharmacy, Science and Technology of Tîrgu Mureș 540139, Tîrgu Mureș, Romania

${ }^{4}$ Department of Toxicology and Biopharmacy, Faculty of Pharmacy, University of Medicine, Pharmacy, Science and Technology of Tîrgu Mureș 540139, Tîrgu Mureș, Romania

${ }^{5}$ Department of Pharmaceutical Industry and Management, Faculty of Pharmacy, University of Medicine, Pharmacy, Science and Technology of Tîrgu Mureș, 540139, Tîrgu Mureș, Romania

${ }^{6}$ Department of Pharmaceutical Chemistry, Faculty of Pharmacy, University of Medicine, Pharmacy, Science and Technology of Tîrgu Mureș

540139, Tîrgu Mureș, Romania
Offering a systematic and multivariate analysis of the analytical procedure, development and validation of HPLC methods using Quality by Design approach are in the limelight of current research trends. A new, experimental design-aided HPLC method for fampridine was developed and preliminarily validated according to current in-force international guidelines for linearity, accuracy, robustness and precision.

The method offers a high throughput sample analysis, with an elution time of 2.9 minutes, and signal detection without excipient interference performed at $262 \mathrm{~nm}$. The method proved to be linear between $1-15 \mu \mathrm{g} \mathrm{mL} L^{-1}\left(R^{2}=0.9996\right)$. The mean recovery was found to be $98.7 \pm 1.9 \%$ in the tested range of $2.5-7.5 \mu \mathrm{g} \mathrm{mL} \mathrm{mL}^{-1}$. Low RSD values ( $<1 \%$ ) were obtained for both model, intra- and inter-day precision. The limit of detection and limit of quantification were 0.24 and $0.78 \mu \mathrm{g} \mathrm{mL}^{-1}$, resp. The method proved to be applicable for active substance assay in a pharmaceutical dosage form.

Keywords: Analytical Quality by Design, fampridine, HPLC

Accepted November 20, 2019

Published online December 10, 2019

Multiple sclerosis (MS) is a chronic neuroinflammatory disease that affects approximately 2.5 million people worldwide. Genetic susceptibility and environmental factors are

\footnotetext{
*Correspondence, e-mail: ibolya.fulop@umfst.ro
} 
probably involved in the onset of the disease, however, the exact cause of MS is still unknown (1). Fampridine (4-aminopyridine, dalfampridine, Fig. 1) is a recently developed therapeutic agent, that complements the currently available disease-modifying treatments by ameliorating the walking impairment, a symptom with high prevalence among MS patients (2). Fampridine is a broad-spectrum voltage-dependent potassium channel blocker first used in electrophysiological studies to determine the role of potassium channels in the generation and propagation of neuronal action potentials $(3,4)$. Following two successful phase-3 clinical trials (5, 6), the US Food and Drug Administration (FDA, 2010) and the European Medicines Agency (EMA, 2011) approved fampridine for the management of walking disability in patients with MS $(7,8)$.<smiles>Nc1ccncc1</smiles>

Fig. 1. Chemical structure of fampridine (4-aminopyridine).

A limited number of analytical methods have been developed for the assay of fampridine. Determination of the active substance and related impurities in the bulk drug or formulations has been achieved using UV spectrophotometry (9) or high-performance liquid chromatography (HPLC) (10-13). Furthermore, liquid chromatography/mass spectrometry (LC-MS) procedures have been developed and applied for the assay of fampridine in biological samples, either individually (14) or simultaneously with other active substances $(15,16)$.

The United States Pharmacopeia's (USP) monograph for dalfampridine (US name for fampridine) describes an HPLC assay procedure that uses as mobile phase methanol and a buffer solution containing octanesulfonic acid sodium salt, ammonium acetate and trimethylamine (17). Thomas et al. (10) used a mobile phase $(\mathrm{pH}=4.00 \pm 0.05)$ of similar composition for the determination of fampridine and potential impurities in the bulk drug. The method described by Babu et al. (11) employs as mobile phase A buffer solution of potassium dihydrogen phosphate and octanesulfonic acid and as mobile phase B mixture of acetonitrile and mobile phase A. Dharani et al. (12) have reported a simpler mobile phase composition, consisting of potassium dihydrogen phosphate solution and acetonitrile with an isocratic elution.

A design of experiments (DoE) approach supports the simultaneous variation of multiple factors at different levels, allowing the detection of both main effects and secondary interactions between the studied factors $(18,19)$. Therefore, DoE provides relevant information with a minimal number of performed experiments (20). Based on the results of these experiments the optimal run conditions can be statistically determined and the outcome of unperformed experiments predicted with high accuracy (21). Pharmaceutical applications of DoE include the development and optimization of single-component $(22,23)$ and multi-component formulations $(24,25)$, as well as the development and validation of analytical methods $(26,27)$. 
The main objective of the present study was to develop and validate a high throughput HPLC method for the assay of fampridine applying DoE using readily available reagents, thus providing a simple and cost-effective alternative to available techniques. Furthermore, we examined the effectiveness of a DoE approach for method development and validation.

\section{EXPERIMENTAL}

\section{Instrumentation}

HPLC determination was carried out using a LiChrosphere ${ }^{\circledR} 60$ RP-select B $5 \mu \mathrm{m}, 250$ $\mathrm{mm} \times 4 \mathrm{~mm}$ column (Merck KGaA, Germany). The components of the Merck Hitachi HPLC system were the following: interface D-7000, quaternary pump L-7100, solvent degasser L-7612, autosampler L-7200, column oven L-7360 and DAD detector L-7455. Specific chromatograms were analysed using the HSM 4.0 software by Merck Hitachi (Merck Hitachi, Japan).

Reagents

Disodium hydrogen phosphate, monobasic potassium phosphate, orthophosphoric acid, sodium hydroxide and HPLC grade methanol $(\mathrm{MeOH})$ were used from Merck (Merck). Gradient grade acetonitrile (ACN) was from VWR International (VWR International, France). Dalfampridine USP reference standard was obtained from USP (USP, Rockville, MD, USA).

The active substance fampridine (FAM) was obtained from Procos (Procos SpA, Italy). Water, purified, was obtained with a Merck Millipore Direct Q, Progard $2^{\circledR}$ system (Merck Millipore, USA).

Selectivity studies were performed based on the qualitative and quantitative composition of the original product, Fampyra $10 \mathrm{mg}$ prolonged-release tablets, using Hypromellose (Dow Chemical, USA), cellulose, microcrystalline (JRS Pharma, Germany), silica, colloidal anhydrous (Evonik Industries, Germany) and magnesium stearate (Faci SpA, Italy) as excipients. The original product was purchased from a local pharmacy.

Preparation of phosphate buffer solution. - Phosphate buffer solution with $\mathrm{pH}=6.8$ was prepared according to the current USP (17).

Preparation of placebo solution (real blank). - The quantities of selected excipients corresponding to one single dose of pharmaceutical formulation were stirred using $900 \mathrm{~mL}$ phosphate buffer, $\mathrm{pH}=6.8$. Ten $\mathrm{mL}$ of the solution was filtered through a $0.45-\mu \mathrm{m}$ Chromafil ${ }^{\circledR}$ Xtra PA-45/25 filters (Macherey-Nagel, Germany) and completed to $1 \mathrm{~L}$ in a volumetric flask with water, obtaining a 1:100 diluted placebo.

Preparation of standard solutions. - The solution of a final concentration of $5 \mu \mathrm{g} \mathrm{mL} \mathrm{L}^{-1}$ was prepared by dissolving $5 \mathrm{mg}$ FAM in $1 \mathrm{~L}$ of 1:100 diluted placebo formulation and completed to the mark with the same solvent.

The starting solution for linearity testing was prepared by dissolving $20 \mathrm{mg}$ FAM in $1 \mathrm{~L}$ of 1:100 diluted placebo formulation and completed to the mark with the same solvent, thus obtaining a final concentration of $20 \mu \mathrm{g} \mathrm{mL} \mathrm{L}^{-1}$. 


\section{Chromatographic conditions}

Chromatograms were recorded under the conditions obtained by in silico method development optimization, using an isocratic mobile phase consisting of MeOH:ACN:PB 65:5:30 $(\%, V / V)\left(\mathrm{PB}-\right.$ phosphate buffer $1 \mathrm{mmol} \mathrm{L}^{-1}, \mathrm{pH}=3.0 \pm 0.01$ adjusted with $\left.\mathrm{H}_{3} \mathrm{PO}_{4}\right)$. The flow rate was set at $1.0 \mathrm{~mL} \mathrm{~min}^{-1}$, using a column temperature of $39.5^{\circ} \mathrm{C}$ and an injection volume of $30 \mu \mathrm{L}$ using the cut method. Analytical signal detection and best chromatogram extraction were recorded at $262 \mathrm{~nm}$.

\section{Method optimization}

Screening experimental design. - The analytical method was optimized by experimental design using the MODDE 12.1 software (Sartorius Stedim Biotech GmbH, Göttingen, Germany). For the best evaluation of the chromatographic method and to profoundly investigate the effects of defined factors on selected responses a full factorial design with three center points was chosen. The included factors: flow rate, column temperature and the proportion of the phosphate buffer, $\mathrm{pH}=3.0$, were investigated at three levels, returning in 30 experiments $\left(3^{3}=27\right.$ in this case, yielding a total of 30 runs including the three center, replicate runs). The level of $\mathrm{ACN}$ was kept at a constant ratio of $5 \%$ throughout the method development and $\mathrm{MeOH}$ proportion was modified according to DoE settings for the percentage of phosphate buffer, $\mathrm{pH}=3.0$. Samples were analysed in three replicate runs, retention time, theoretical plate number and tailing factor were recorded as responses (Table I).

Model fitting and analysis of the experimental design. - The experimental model was fitted using the partial least squares (PLS) method and the significance was tested by ANOVA F-test and its test for lack-of-fit. Further analysis included the evaluation of model performance indicators characterizing the factor-response interactions. Generally, a model is

Table I. Factors and responses defined in the present screening experimental design

\begin{tabular}{llll}
\hline Selected factors for the experimental design & & & \\
\hline Level applied & -1 & 0 & 1 \\
Flow rate $\left({\left.\mathrm{mL} \mathrm{min}^{-1}\right)}^{\text {Column temperature }\left({ }^{\circ} \mathrm{C}\right)}\right.$ & 0.50 & 1.00 & 1.25 \\
PB proportion in mobile phase $(\%)^{\mathrm{a}}$ & 20 & 30 & 40 \\
\hline Responses followed for experimental design assessment & 30 & 50 & 70 \\
\hline Requirement & Min & Target & Max \\
Retention time $(\mathrm{min})$ & 2 & 3 & 5 \\
Theoretical plate number $(N)^{\mathrm{b}}$ & 2000 & - & - \\
Tailing factor & 0.8 & 1.2 & 2.0 \\
\hline
\end{tabular}

a $\mathrm{PB}$ - phosphate buffer, $\mathrm{pH}=3.0$.

${ }^{\mathrm{b}}$ Defined per column length. 
considered adequate if the goodness of fit $\left(R^{2}\right)$ and goodness of predictability $\left(Q^{2}\right)$ tend to 1.0, but are greater than 0.5 and the difference between the two indicators does not exceed 0.2-0.3. Model validity, describing the suitability of the selected method for the analysis of the design should return values greater than 0.25 . Finally, model reproducibility offers information about the controllability of the proposed method and is considered acceptable if the values are situated above 0.5 . In the case of reproducibility, excessively high numerical values $(>0.99)$ indicate an infinitesimal pure error inside the model and is generally observed in highly controlled methods, e.g., HPLC determinations. As model validity is closely related to the pure error, low values $(<0.25)$ might be observed when high reproducibility is attained, and thus the design is considered as acceptable in these particular cases as well $(19,28)$. Furthermore, factor-response interactions were also evaluated by the coefficient plot analysis, where model refinement was attained by removing non-significant model terms $(p>0.05)$.

Optimization. - After model fitting and refinement, an optimizer run was carried out based on the parameter settings provided by the software. The optimizer run was injected five times and the obtained results were compared with the predicted results by the computational program using the prediction spreadsheet option.

\section{Preliminary validation of the method after in silico optimization}

Linearity. - Method linearity was tested in the range of $1-15 \mu \mathrm{g} \mathrm{mL}^{-1}$ through six sam-

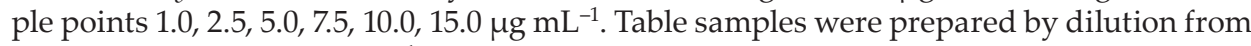
a stock solution of $20 \mu \mathrm{g} \mathrm{mL} \mathrm{m}^{-1}$. The determination was repeated five times for each concentration level.

Model selectivity. - Selectivity studies were conducted by recording the chromatograms of individual excipient samples, placebo formulation and placebo spiked with FAM prepared under the same conditions.

Model accuracy. - Method recovery was evaluated at three points (50, 100 and $150 \%)$ of the target concentration. Individual samples were prepared with FAM-spiked placebo and repeated three times for each determination.

Robustness. - Method robustness was tested by applying a separate experimental design (Plackett-Burman model, fitted with the multiple linear regression method) (19). The model offers the possibility to assess the linear factor-response relationship, but no factor interactions can be evaluated by using the disclosed experimental model. In the case of robustness testing using experimental designs, four general outcomes (limiting cases) of the obtained results are defined. Amongst the various outcomes of the fitted and refined Plackett-Burman model, the limiting case of inside specification limits, but non-significant relationship is considered as an optimal result for robustness testing. For the robustness testing the same factors were defined as described in the previous experimental design with the following settings: flow rate $0.95-1.05 \mathrm{~mL} \mathrm{~min}^{-1}$, column temperature $37.5-41.5^{\circ} \mathrm{C}$ and the proportion of the phosphate buffer, $\mathrm{pH}=3.0$, between 28 and $32(\%, V / V)$. The experimental design returned 11 experimental runs.

Furthermore, method robustness was tested for individual changes in detection wavelength at 260 and $264 \mathrm{~nm}$. 
Model precision. - Placebo samples spiked with $5 \mathrm{mg}$ of FAM were prepared on the same day by the same analyst and on two different days by two analysts. The analysis was carried out on six replicate samples for each determination. Instrument precision was tested on five replicate injections of the same sample.

Limit of detection (LOD) and limit of quantification (LOQ). - LOD and LOQ values were assessed at a 3:1 and 10:1 signal-to-noise ratio for six and three replicate determinations, resp.

\section{Assay from the pharmaceutical dosage form}

Ten tablets of Fampyra $10 \mathrm{mg}$ prolonged-release tablets were pulverized and $200 \mathrm{mg}$ of sample, corresponding to $5 \mathrm{mg}$ FAM was admixtured with $500 \mathrm{~mL}$ phosphate buffer, $\mathrm{pH}$ $=6.8$, sonicated in an ultrasound bath for $15 \mathrm{~min}$ and completed with the same solvent to $1 \mathrm{~L}$ in a volumetric flask. The final sample was filtered through a $0.45-\mu \mathrm{m}$ Chromafil ${ }^{\circledR}$ Xtra PA-45/25 filter discarding the first $5 \mathrm{~mL}$ of filtrate. Active substance content was analysed from three individual samples.

\section{Statistical analysis}

Statistical analyses were carried out using the Minitab 17 software (Minitab Inc., State College, PA, USA) and Statistica 8.0 software (StatSoft, Tulsa, OK, USA).

Possible aberrant values in experimental design were detected and excluded using the Grubb's test for outliers at $\alpha=0.05$ significance level $(p<0.05)$.

Shapiro-Wilk's test was used for the assessment of the normal distribution of the residuals, at a $95 \%$ confidence interval. The null hypothesis, that the residuals do not follow a normal distribution, is rejected if $W$ for FAM is greater than the critical tabulated value for the number of observations at $\alpha=0.05$ and simultaneously $p>0.05$. Furthermore, the plot of response values vs. corresponding concentrations was calculated with an acceptance limit of $2.5 \%$ in comparison to target level response factor in the range of $50-150 \%$ and $5.0 \%$ in the range of tested linearity. ANOVA $F$-test and its test for lack-of-fit were used for the significance testing of the calibration curve $(\mathrm{CI}=95 \%)$. In order to assess the capability of the process to comply within specification limits, process capability $\left(C_{\mathrm{pk}}\right)$ was calculated for the plot of response values $v$ s. corresponding concentrations at $\pm 10 \%$ specification limits (acceptance criteria: $C_{\mathrm{pk}}>1.33$ ).

In order to statistically assess the variability of the sets of the results obtained for intra- and inter-day model precision, Student's $t$-test was used by setting the significance level at $\alpha=0.05$.

\section{RESULTS AND DISCUSSION}

\section{Screening experimental design}

Experiments no. 14 and 15 were excluded from DoE analysis due to inappropriate chromatographic results. Grubb's test performed after the exclusion of the aforementioned experiments did not result in the detection of outlier values. The experimental results are presented in Table II. 


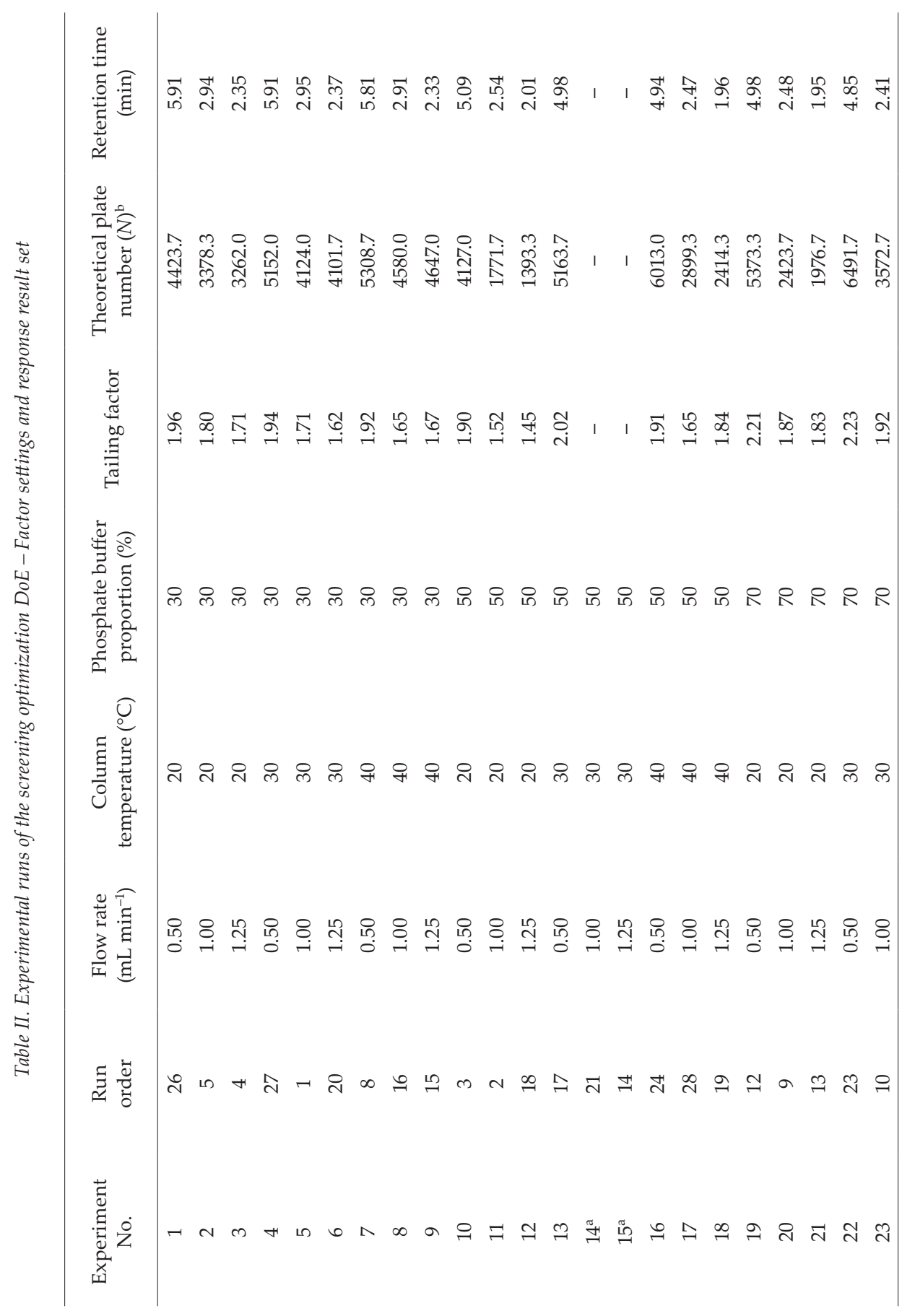



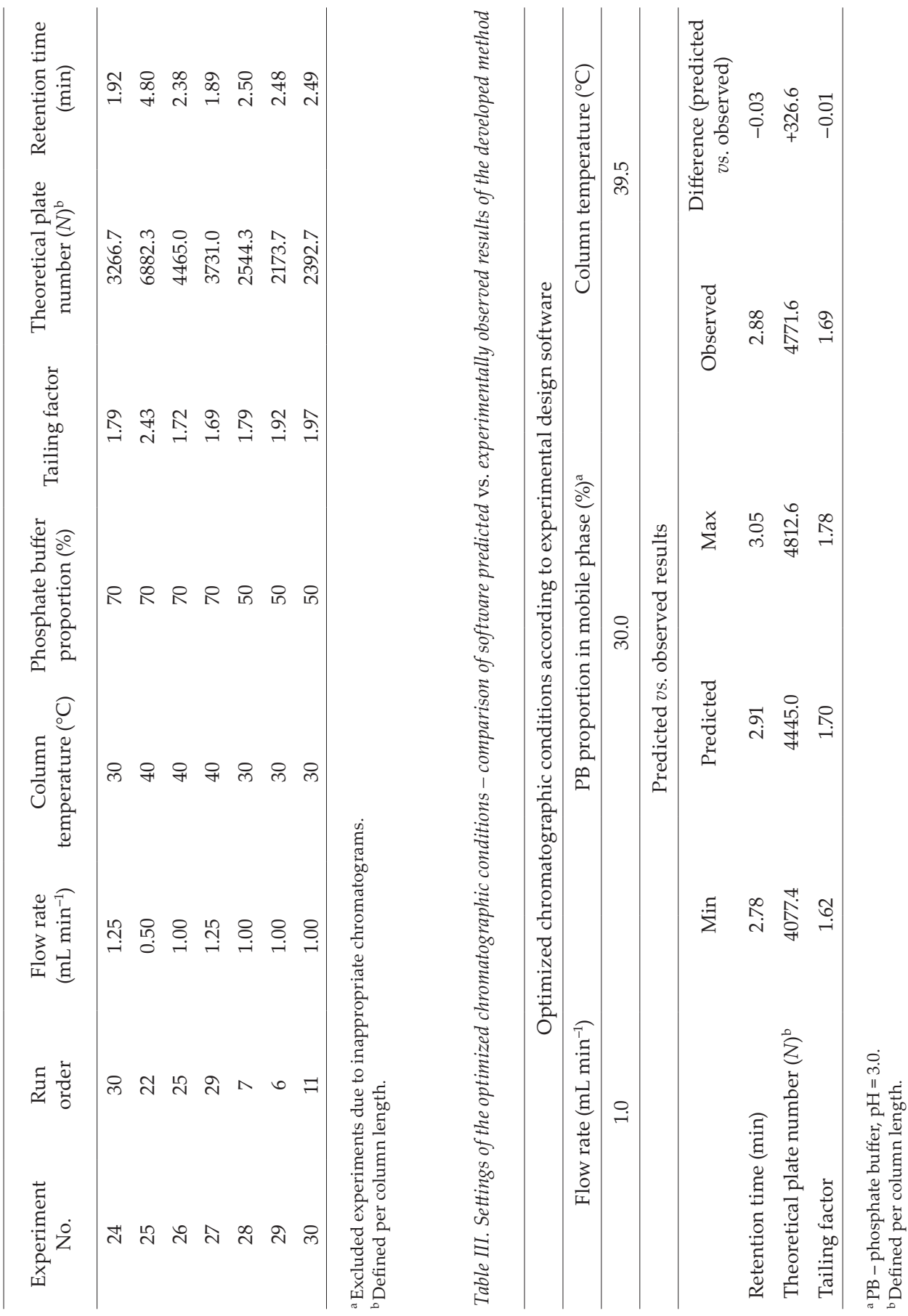
Significant regression models $(p<0.05)$ were obtained in the case of all three studied responses (retention time, theoretical plate number and tailing factor); lack-of-fit was only observed in the case of retention time $(p=0.003)$. In the latter case, low model validity is explained by the high reproducibility $(0.99995)$ of the replicate runs. The identical results obtained for retention time under the replicate runs, as desired in HPLC method development, leads to a low pure error inside the model, or namely high reproducibility. Usually, in this case the model error is substantially greater than the pure error, resulting in a higher lack-of-fit, which in turn persuades in low model validity. The analysis of the model returned good model performance indicator values (Fig. 3a), as $R^{2}$ and $Q^{2}$ values are situated between $0.63-0.98$ and $0.61-0.97$, resp. The difference between the values of goodness of fit $\left(R^{2}\right)$ and goodness of predictability $\left(Q^{2}\right)$ does not exceed the requirement of $0.2-0.3$. The model validity for tailing factor and theoretical plate number is above the minimum threshold of 0.25 , with values of 0.75 and 0.61 , resp. The reproducibility values of the responses are all superior to the minimal criteria of 0.5 , ranging from 0.80 to 0.99 .

The analysis of the coefficient plots (Fig. 3b) revealed that retention time is strongly influenced by the flow rate and phosphate buffer proportion and shows a negative correlation with the flow rate and the proportion of the buffer in the mobile phase. Similarly, in the case of the tailing factor, the flow rate has a negative impact on response results, whilst in terms of buffer concentration a positive correlation was found. The theoretical plate number is influenced in an identical manner by the flow rate as observed in the previous cases. Contrarily to these, in the case of theoretical plate number a strong influence by column temperature can be observed, whilst the effect of the phosphate buffer portion is negligible. Although indicating a curved surface inside the model, the detected factor interactions (flow $\times \mathrm{PB}$ ) and quadratic terms (flow $\times$ flow, $\mathrm{PB} \times \mathrm{PB}$ ) for retention time and theoretical plate number were retained, as deletion of these particular influencing factors would result in performance model indicator deterioration.

Optimizer run. - Based on the fitted and refined experimental design, a placebo sample spiked with $5 \mathrm{mg}$ FAM was injected five times using the factor settings offered by the software and the results were compared with those predicted by the computational design. The optimized factor settings and comparison of the predicted $v$ s. observed result sets are presented in Table III.

Under the set of experimental conditions (flow rate $1.0 \mathrm{~mL} \mathrm{~min}^{-1}$, column temperature $39.5^{\circ} \mathrm{C}$ and $30 \% \mathrm{~PB}$ ) the prediction of the program proved to be reliable, as a sample retention time of $2.88 \mathrm{~min}$ is marginally inferior to the foreseen $2.91 \mathrm{~min}$ and a peak tailing of 1.69 is properly close to the predicted 1.70 . Regarding the values of the theoretical plate number, a higher value was observed in comparison to the predicted one, but still lying within the confidence interval provided by the software. A typical chromatogram under the optimized chromatographic conditions is presented in Fig. $2 \mathrm{~b}$.

\section{Model validation results}

Selectivity. - Under proposed settings, FAM can be identified as a sharp, well-defined peak $262 \mathrm{~nm}$, from both the standard solution and pharmaceutical dosage form, in comparison to placebo formulation (see Figs. 2a-c); no interference of the excipients can be observed (see Fig. 2a).

Linearity. - The method proved to be linear in the tested range of $1-15 \mu \mathrm{g} \mathrm{mL}^{-1}$, with a coefficient of determination of $R^{2}=0.9996$. The Shapiro-Wilk's test for the normal distribu- 
a)

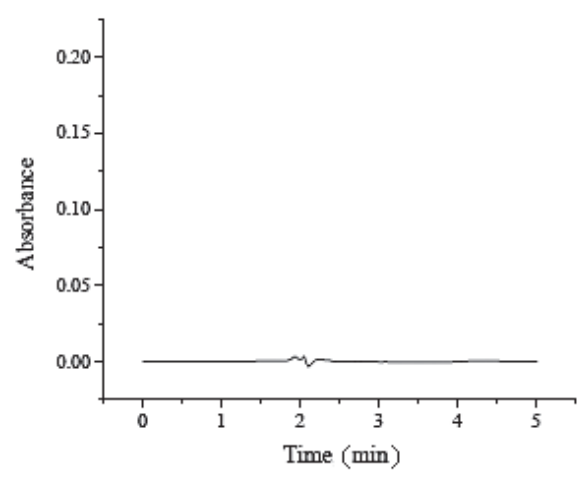

b)

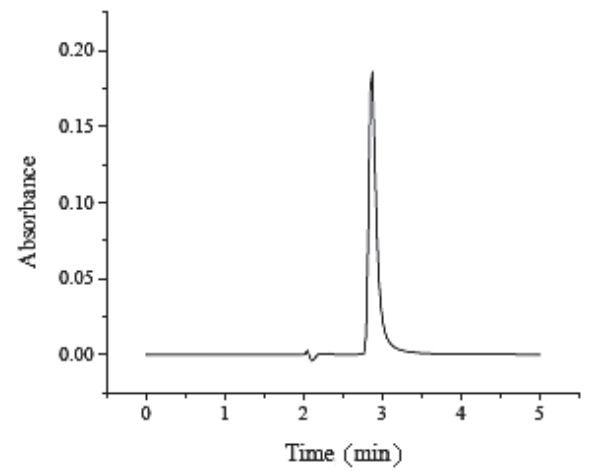

c)

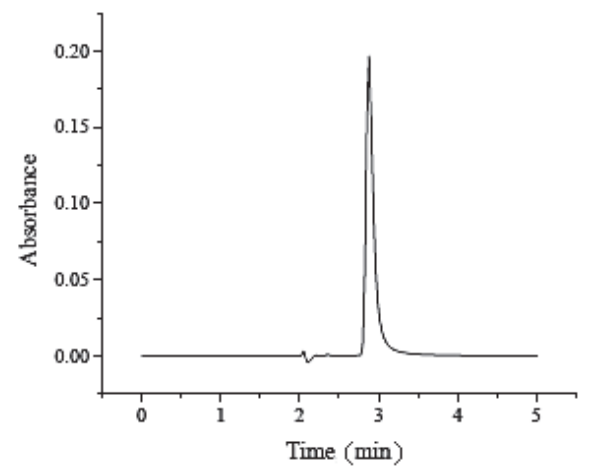

Fig. 2. a) Chromatogram of placebo sample, b) typical chromatogram of placebo spiked with fampridine $\left.\left(5 \mu \mathrm{g} \mathrm{mL}^{-1}\right), \mathrm{c}\right)$ chromatogram of a real sample obtained from the original commercial product $\left(5 \mu \mathrm{g} \mathrm{mL} \mathrm{L}^{-1} \mathrm{FAM}\right)$.

tion of the residuals was passed, as the obtained $\mathrm{W}_{\mathrm{FAM}}=0.866$ was greater than the critical threshold of $\mathrm{W}_{\text {CRIT. }}=0.788(n=6, \alpha=95 \%), p=0.212$. The ANOVA F-test revealed a significant relationship between the predictors and responses $(p<0.05)$ and no lack-of-fit was observed $(p>0.05)$. The capability analysis of the plot of response values vs. concentration resulted in a $C_{\mathrm{pk}}=1.72$, indicating that the model is well controlled within the proposed linearity range. Statistical results are given in Table IV.

Robustness. - The method proved to be robust in the tested range as minor changes in the chromatographic conditions did not influence the detectability of the analyte (Table V). This is also supported by the results of the Plackett-Burman experimental design, where no significant relationship was found between the factors and responses in the case of tailing factor and theoretical plate number $\left(R^{2}<0.5\right)$. Concurrently, the low goodness of predictability values indicates that the model could not detect remarkable modifications in response values that are due to minor changes in factor settings. 
Table IV. Model analytical merits of the developed HPLC method

\begin{tabular}{|c|c|c|}
\hline Parameter & Result & Statistical result \\
\hline Linearity $\left(\mu \mathrm{g} \mathrm{mL} L^{-1}\right)$ & $1-15 \mu \mathrm{g} \mathrm{mL} L^{-1}$ & $\begin{array}{c}R^{2}=0.9996 \\
W_{\text {FAM }}=0.866^{\mathrm{a}}(p=0.212) \\
F=22,132.69(p<0.05)^{\mathrm{b}} \\
F=2.35(p>0.05)^{\mathrm{c}} \\
C_{\mathrm{pk}}=1.72^{\mathrm{d}}\end{array}$ \\
\hline Accuracy (\%) & $98.7 \pm 1.9$ & $R^{2}=0.9998$ \\
\hline $\begin{array}{l}\text { Intraday precision } \\
(\text { RSD, \% })^{\mathrm{e}, \mathrm{f}}\end{array}$ & $0.4-0.7$ & $\begin{array}{c}t_{<\text {analyst } 1 \text { day } 1 \text { vs. analyst } 2 \text { day } 1>}=1.548 \\
p=0.153\end{array}$ \\
\hline $\begin{array}{l}\text { Inter-day precision } \\
\quad(\text { RSD }, \%)^{\mathrm{e}, \mathrm{f}}\end{array}$ & 0.5 & $\begin{array}{c}t_{<\text {analyst } 1 \text { day } 1 \text { vs. analyst } 1 \text { day } 2>}=1.682 \\
p=0.123 \\
t_{<\text {analyst } 2 \text { day } 1 \text { vs. analyst day } 2>}=0.237 \\
p=0.816\end{array}$ \\
\hline $\begin{array}{l}\text { Instrument precision } \\
(\text { RSD, \% })^{g}\end{array}$ & 0.5 & - \\
\hline$L O D\left(\mu \mathrm{g} \mathrm{mL}^{-1}\right)$ & 0.24 & - \\
\hline$L O Q\left(\mu g \mathrm{~mL}^{-1}\right)$ & 0.78 & - \\
\hline Assay from tablets $(\%)^{\mathrm{h}, \mathrm{i}}$ & 100.3 & - \\
\hline
\end{tabular}

${ }^{\mathrm{a}} W_{\text {CRIT. }}=0.788-$ critical tabulated value of Shapiro-Wilk's test.

${ }^{\mathrm{b}}$ Critical tabulated value of ANOVA $F$-test, $F=4.20$.

${ }^{\mathrm{c}}$ Critical tabulated value of ANOVA $F$-test for lack-of-fit, $F=2.70$.

${ }^{\mathrm{d}}$ Requirement, $C_{\mathrm{pk}}>1.33$.

${ }^{\text {e }}$ Critical value of $t=2.228, \mathrm{df}=10$.

${ }^{\mathrm{f}} n=6,{ }^{\mathrm{g}} n=5,{ }^{\mathrm{h}} n=3$.

${ }^{\mathrm{i}}$ Compliance with the label claim.

In contrast to the screening experimental design, in the case of the Plackett-Burman model higher model validity values are attained (Fig. 3c), whereas in the case of retention time a negative to positive switch is observed, indicating that the model is valid in the proposed testing range.

The analysis of the coefficient plots (Fig. 3d) shows that the theoretical plate number and tailing factor are not influenced in a significant manner by any of the defined factors. In contrast, retention time is strongly influenced by the flow rate and the proportion of the phosphate buffer in the mobile phase, both having a negative correlation with the mentioned response. These tendencies are similar to those observed in the screening experimental model. The existence of strong factor to response correlations explains the higher $R^{2}$ and $Q^{2}$ values, since, as expected, minor changes in flow rate and mobile phase composition have a slight effect on the retention of the active substance to the column's stationary phase, thus modifying retention time in a systematic manner.

Negligible changes in detection wavelength $( \pm 2 \mathrm{~nm})$ did not influence the selected responses in a critical manner, resulting in a tailing factor of 1.66 and a theoretical plate number of 4712 in both cases, and a recovery of 99.5 and $99.9 \%$ at 260 and $264 \mathrm{~nm}$, resp. 


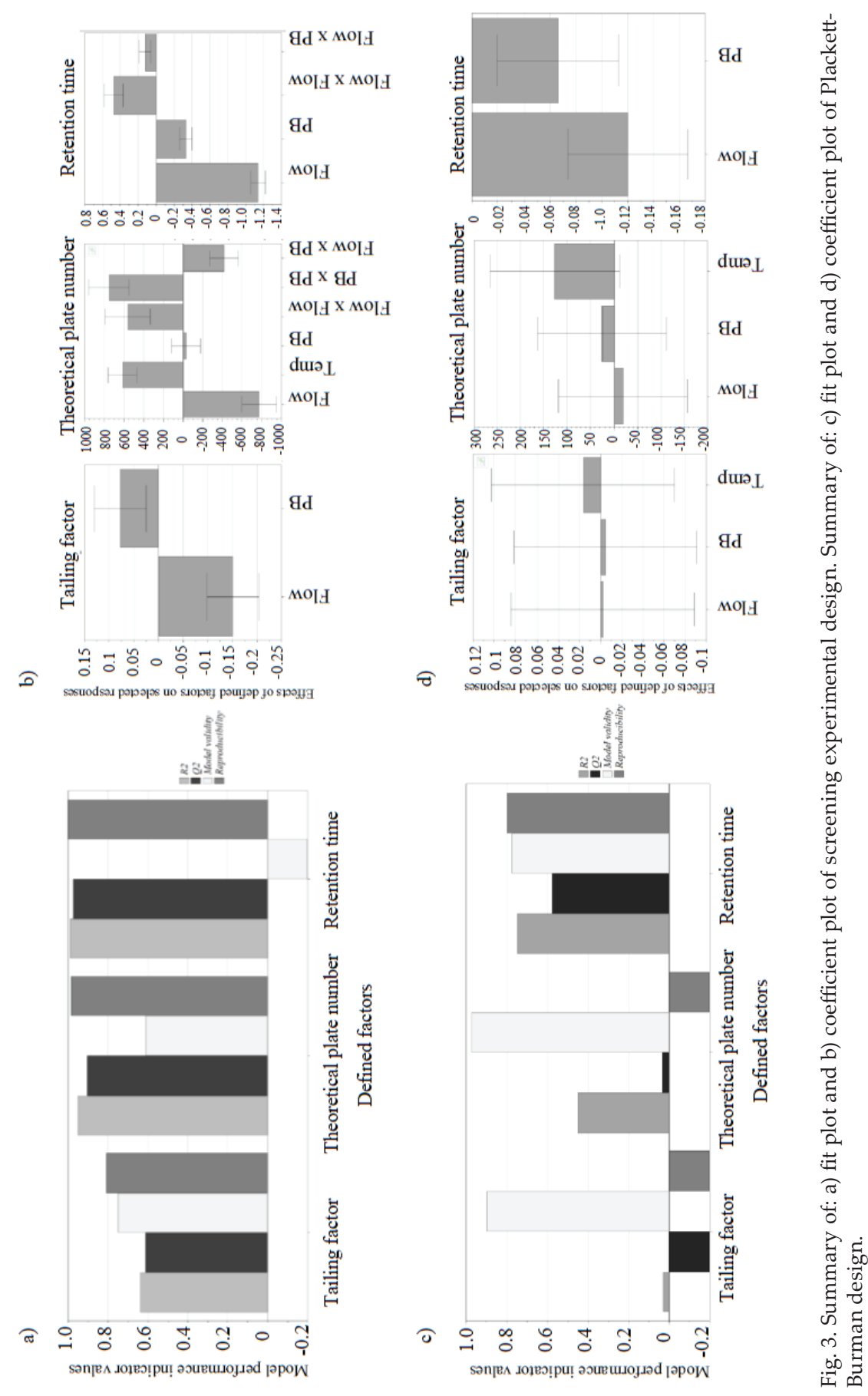


Accuracy. - The mean recovery of the method was $98.7 \%$, value lying in-between 95.8 and $101.8 \%$ for the $50-150 \%$ interval (Tables IV and VI). Also, the linearity of the spiked samples shows a good linear correlation, with $R^{2}=0.9998$.

Precision. - The performed Student's $t$-test revealed that there is no statistical difference between the sample set prepared by the same analyst on the same day (intra-day precision) and between the two analyst's sample set prepared on two different days (inter-day precision). The obtained results showed low variability between the samples, with an RSD of $0.6 \%$ (100.5-103.3 \%). An instrument precision with an RSD of $0.5 \%$ (99.9-101.3 \%) was obtained after five successive injections of the same sample solution (Tables IV and VI).

Limit of detection (LOD) and limit of quantification (LOQ). - The limits of detection and quantification of the developed method are 0.24 and $0.78 \mu \mathrm{g} \mathrm{mL} \mathrm{L}^{-1}$, resp.

Assay of the pharmaceutical dosage form

Three replicate samples yielded an average recovery of $100.6 \%$ (RSD $=0.7 \%$ ) from FAM tablet formulation (Tables IV and VI) (Fig. 2c).

\section{Method benefits}

The proposed analytical method offers a high throughput analysis for FAM and was aimed to be useful in the assay of the active pharmaceutical ingredient from tablets and

Table V. Experimental runs of the robustness testing DoE - Factor settings and response result set

\begin{tabular}{ccccccc}
\hline $\begin{array}{c}\text { Experiment } \\
\text { no. }\end{array}$ & $\begin{array}{c}\text { Flow rate } \\
\left(\mathrm{mL} \mathrm{min}^{-1}\right)\end{array}$ & $\begin{array}{c}\text { Column } \\
\text { temperature } \\
\left({ }^{\circ} \mathrm{C}\right)\end{array}$ & $\begin{array}{c}\text { Phosphate } \\
\text { buffer } \\
\text { proportion }(\%)\end{array}$ & $\begin{array}{c}\text { Retention } \\
\text { time }(\mathrm{min})\end{array}$ & $\begin{array}{c}\text { Tailing } \\
\text { factor }\end{array}$ & $\begin{array}{c}\text { Theoretical } \\
\text { plate number } \\
(\mathrm{N})^{\mathrm{a}}\end{array}$ \\
\hline 1 & 1.05 & 28 & 37.5 & 2.85 & 1.81 & 4847.0 \\
2 & 1.05 & 32 & 37.5 & 2.85 & 1.81 & 4847.0 \\
3 & 1.05 & 32 & 41.5 & 2.72 & 1.90 & 5003.0 \\
4 & 0.95 & 32 & 41.5 & 3.01 & 1.79 & 5247.0 \\
5 & 1.05 & 28 & 41.5 & 2.92 & 1.80 & 5078.0 \\
6 & 0.95 & 32 & 37.5 & 2.97 & 1.81 & 4857.0 \\
7 & 0.95 & 28 & 41.5 & 3.18 & 1.90 & 5032.5 \\
8 & 0.95 & 28 & 37.5 & 3.14 & 1.84 & 4792.5 \\
9 & 1.00 & 30 & 39.5 & 2.87 & 1.71 & 4991.0 \\
10 & 1.00 & 30 & 39.5 & 2.88 & 1.59 & 4772.0 \\
11 & 1.000 & 30 & 39.5 & 2.91 & 1.82 & 4576.0 \\
\hline Average & & & 2.93 & 1.79 & 4913.0 \\
\hline Difference to predicted optimizer results & & +0.02 & +0.09 & +468.0 \\
\hline Difference to observed optimizer results
\end{tabular}

${ }^{a}$ Defined per column length. 
Table VI. Results obtained for model studies of accuracy, precision and pharmaceutical dosage form

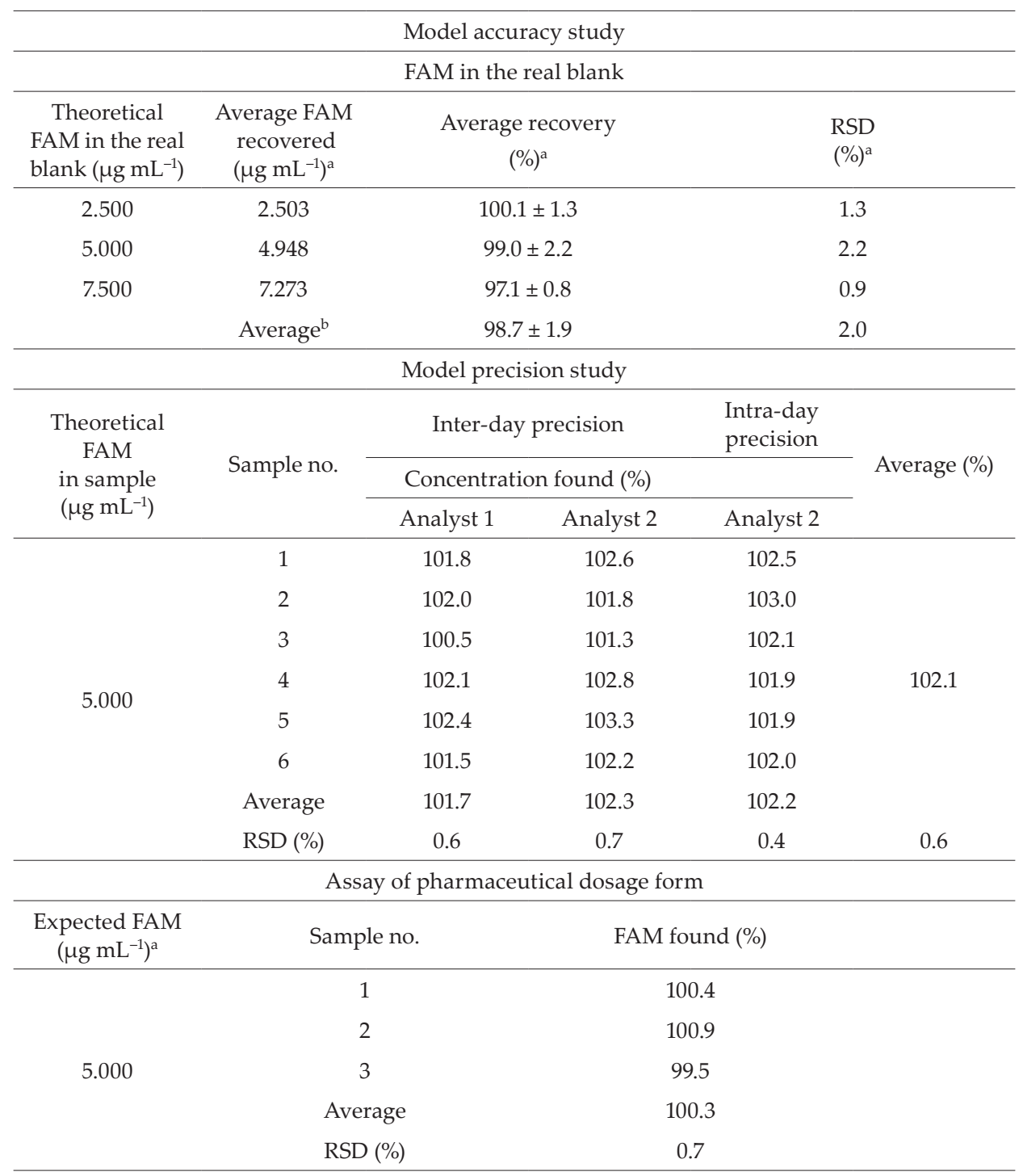

${ }^{\mathrm{a}} n=9,{ }^{\mathrm{b}} n=27,{ }^{\mathrm{c}}$ Based upon label claim.

dissolution profile samples. When compared to the already available methods (see Table VII) that aimed only the assay of the active substance (12), the novel method has a lower linearity range of $1-15 \mu \mathrm{g} \mathrm{mL} \mathrm{L}^{-1}$ vs. $20-80 \mu \mathrm{g} \mathrm{mL}-1$. Comparing the other analytical validation parameters, the novel method surpasses the currently accessible method (12), espe- 


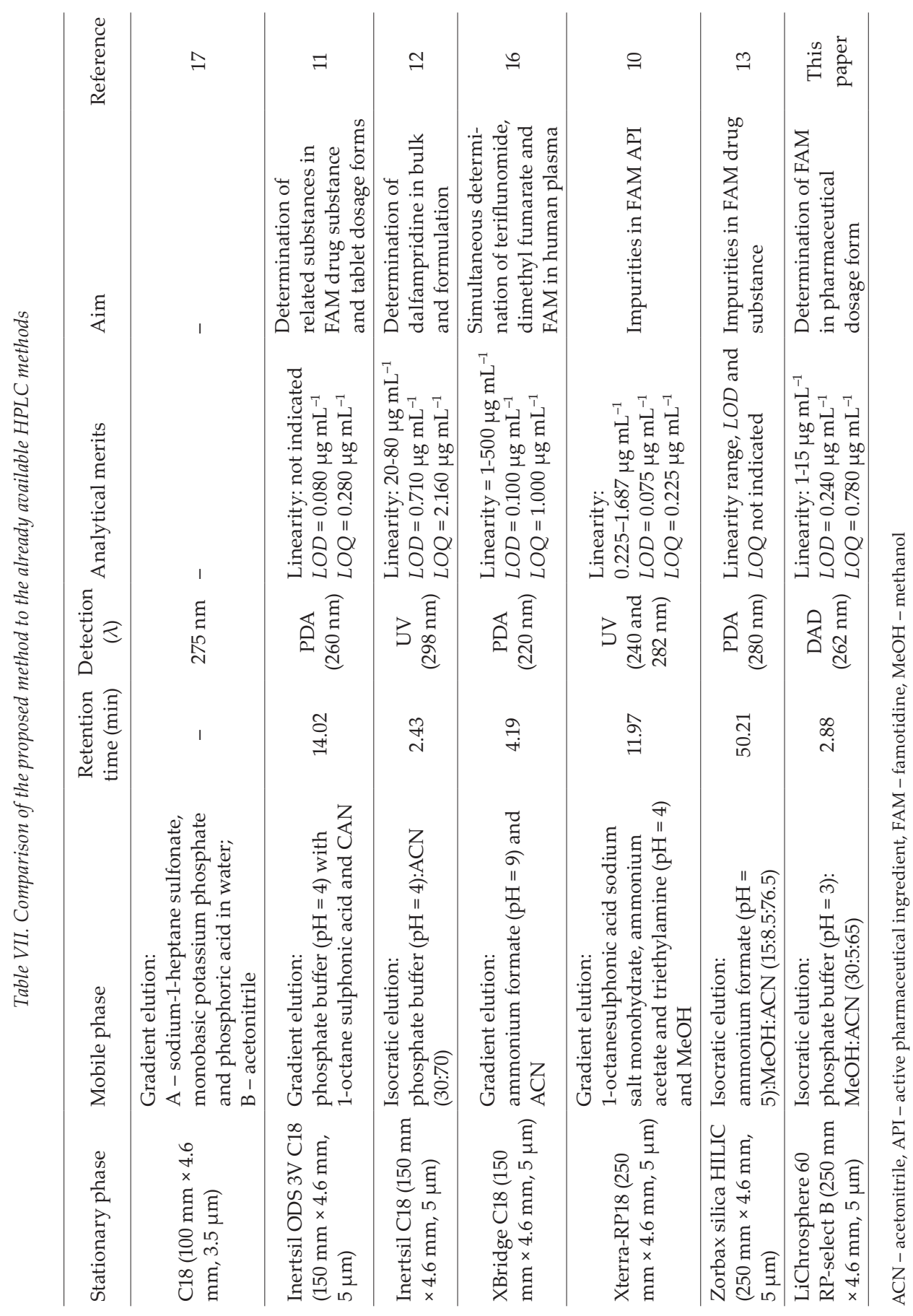


cially in terms of $L O D$ and $L O Q$ of $0.24 \mu \mathrm{g} \mathrm{mL}^{-1}$ vs. $0.71 \mu \mathrm{g} \mathrm{mL} \mathrm{m}^{-1}$ and $0.78 \mu \mathrm{g} \mathrm{mL} \mathrm{L}^{-1}$ vs. 2.16 $\mu \mathrm{g} \mathrm{mL} \mathrm{L}^{-1}$, resp. Furthermore, in terms of retention time, the proposed method performs better or equivalently in contrast to other analytical reports. Taking into consideration the FDA requirement (29) for the volume of dissolution media $(900 \mathrm{~mL})$ and the amount of the active substance $(10 \mathrm{mg})$, a general concentration span between $1.11 \mu \mathrm{g} \mathrm{mL} \mathrm{m}^{-1}(10 \%$ of active substance dissolved) and $11.11 \mu \mathrm{g} \mathrm{mL} \mathrm{m}^{-1}$ (100\% of active substance dissolved) would be obtained at specified sampling times $(0.5-12 \mathrm{~h})$. As method validation was carried out nearby the aforementioned concentration range, the novel method might perform worthier when compared with other methods and is applicable without time-consuming sample preparation. Furthermore, the developed method might be considered as a surrogate for the available compendial method, having its benefit in the simpler and more available mobile phase components.

\section{CONCLUSIONS}

The developed method offers high throughput, cost-effective sample analysis from both bulk samples and pharmaceutical dosage form. The applied computational method development offers the possibility to collect in silico information regarding the chromatographic particularities following modifications in factor settings. The newly proposed method for the assay of fampridine was preliminarily validated as per the currently available international guidelines and fulfilled the model validation requirements for linearity, accuracy, robustness and precision. Furthermore, the development and validation of the present method employing Quality by Design approach support the essentiality of software-controlled methodology in modern pharmaceutical research and development.

Acknowledgements. - The current research was partially funded by the Transylvanian Museum Society, Department of Medicine and Pharmacy and the University of Semmelweis, Faculty of Pharmacy, grant no. 84.6/2017/P.2/EMEOGYSZ. B. Kovács and F. Boda are awarded with a Collegium Talentum scholarship and thank for the support.

The Author's due to confidential/possible conflicted reasons regarding the original product would not like to disclose the exact quantities used for placebo formulation.

\section{REFERENCES}

1. C. A. Dendrou, L. Fugger and M. A. Friese, Immunopathology of multiple sclerosis, Nat. Rev. Immunol. 15 (2015) 545-558; https://doi.org/10.1038/nri3871

2. A. Lugaresi, Pharmacology and clinical efficacy of dalfampridine for treating multiple sclerosis, Expert Opin. Drug Metab. Toxicol. 11 (2014) 295-306; https://doi.org/10.1517/17425255.2015.993315

3. S. Weir, R. Torkin and H. R. Henney, Pharmacokinetic profile of dalfampridine extended release: clinical relevance in patients with multiple sclerosis, Curr. Med. Res. Opin. 29 (2012) 1627-1636; https://doi.org/10.1185/03007995.2012.749221

4. W. A. Coetzee, Y. Amarillo, J. Chiu, A. Chow, D. Lau, T. McCormack, H. Moreno, M. S. Nadal, A. Ozaita, D. Pountney, M. Saganich, E. V. S. de Miera and B. Rudy, Molecular diversity of $\mathrm{K}^{+}$channels, Ann. N. Y. Acad. Sci. 868 (1999) 233-255.

5. A. D. Goodman, T. R. Brown, L. B. Krupp, R. T. Schapiro, S. R. Schwid, R. Cohen, L. M. Marinucci and A. R. Blight, Sustained-release oral fampridine in multiple sclerosis: a randomised, doubleblind, controlled trial, Lancet 373 (2009) 732-738; https://doi.org/10.1016/S0140-6736(09)60442-6 
6. A. D. Goodman, T. R. Brown, K. R. Edwards, L. B. Krupp, R. T. Schapiro, R. Cohen, L. N. Marinucci and A. R. Blight, A phase 3 trial of extended release oral dalfampridine in multiple sclerosis, Ann. Neurol. 68 (2010) 494-502; https://doi.org/10.1002/ana.22240

7. US Food and Drug Administration (FDA), Center for Drug Evaluation and Research (CDER), Application Number 22-250s000, Approval Letter, FDA, Silver Spring (MD) 2010; https://www.accessdata.fda.gov/drugsatfda_docs/nda/2010/022250s000_Approv.pdf; last access date June 15, 2019

8. European Medicines Agency (EMA), Committee for Medicinal Products for Human Use (CHMP), Summary of Opinion (Initial Authorisation) for Fampyra (Fampridine), May 2011; https://www.ema. europa.eu/en/documents/smop-initial/chmp-summary-positive-opinion-fampyra_en.pdf; last access date June 18, 2019

9. V. K. Redasani, G. L. Shaikh and S. S. Surana, Development and validation of spectroscopic methods for the estimation of dalfampridine in bulk and in tablet formulation, Anal. Chem. Indian J. 14 (2014) $37-41$.

10. S. Thomas, S. Shandilya, A. Bharti and A. Agarwal, A stability indicating simultaneous dual wavelength UV-HPLC method for the determination of potential impurities in fampridine active pharmaceutical ingredient, J. Pharm. Biomed. Anal. 58 (2012) 136-140; https://doi.org/10.1016/j. jpba.2011.09.009

11. C. Babu, K. N. Rao, N. Devanna and K. S Reddy, Development and validation of stability indicating reversed phase high performance liquid chromatographic method for the determination of related substances in fampridine drug substance and tablet dosage forms, Asian J. Pharm. Clin. Res. 10 (2017) 334-338.

12. N. R. Dharani, K. Padmini and S. Sumakala, Stability indicating RP-HPLC method development and validation for estimation of dalfampridine in its bulk and formulation, Int. J. Adv. Res. 4 (2016) 184-191; https://doi.org/10.21474/IJAR01/1471

13. M. Jain, V. Srivastava, R. Kumar, V. Dangi, S. G. Hiriyanna, A. Kumar and P. Kumar, Determination of five potential genotoxic impurities in dalfampridine using liquid chromatography, J. Pharm. Biomed. Anal. 133 (2017) 27-31; https://doi.org/10.1016/j.jpba.2016.10.013.

14. W. Smith, S. Swan, T. Marbury and H. Henney, Single-dose pharmacokinetics of sustained-release fampridine (fampridine-SR) in healthy volunteers and adults with renal impairment, J. Clin. Pharmacol. 50 (2010) 151-159; https://doi.org/10.1177/0091270009344857

15. A. Suneetha and R. K. Raja, High-throughput liquid chromatography tandem mass spectrometry method for simultaneous determination of fampridine, paroxetine, and quinidine in rat plasma: Application to in vivo perfusion study, J. Food. Drug Anal. 24 (2016) 866-875; https://doi.org/10.1016/j. jfda.2016.03.004

16. A. Suneetha and R. K. Raja, Comparison of LC-UV and LC-MS methods for simultaneous determination of teriflunomide, dimethyl fumarate and fampridine in human plasma: application to rat pharmacokinetic study, Biomed. Chromatogr. 30 (2016) 1371-1377; https://doi.org/10.1002/ bmc.3694

17. United States Pharmacopeia/National Formulary (USP 42-NF 37), United States Pharmacopeial Convention, Rockville (MD) 2017; last access date November 1, 2019

18. A. Dean, D. Voss and D. Draguljic, Design and Analysis of Experiments, $2^{\text {nd }}$ ed., Springer International Publishing, Cham 2017.

19. L. Eriksson, E. Johansson, N. Kettaneh-Wold, C. Wilkström and S. Wold, Design of Experiments Principles and Applications, $3^{\text {rd }}$ ed., MKS Umetrics AB, Umeå 2008.

20. A. L. Vonica-Gligor, I. Tomuță I and S.E. Leucuța, Piecewise function parameters as responses of the design of experiment in the development of a pulsatile release chronopharmaceutical system, Acta Pharm. 66 (2016) 173-189; https://doi.org/10.1515/acph-2016-0025

21. A. Ćurić, R. Reul, J. Möschwitzer and G. Fricker, Formulation optimization of itraconazole loaded PEGylated liposomes for parenteral administration by using design of experiments, Int. J. Pharm. 448 (2013) 189-197; https://doi.org/10.1016/j.ijpharm.2013.03.029 
22. C. Saroja and P. K. Lakshmi, Formulation and optimization of fenofibrate lipospheres using Taguchi's experimental design, Acta Pharm. 63 (2013) 71-83; https://doi.org/10.2478/acpb-2013-0008

23. B. Sylvester, A. Porfire, D. M. Muntean, L. Vlase and I. Tomuță, Formulation optimization of pravastatin loaded long-circulating liposomes using a design of experiments, Farmacia 64 (2016) $449-458$

24. Z. I. Szabó, B. Székely-Szentmiklósi, B. Deák, I. Székely-Szentmiklósi, B. Kovács, K. Zöldi and E. Sipos, Study of the effect of formulation variables on the characteristics of combination tablets containing enalapril maleate and indapamide as active substances using experimental design, Acta Pharm. 66 (2016) 191-206; https://doi.org/ 10.1515/acph-2016-0019

25. L. R. Tefas, B. Sylvester, I. Tomuță, A. Sesarman, E. Licarete, M Banciu and A. Porfire, Development of antiproliferative long-circulating liposomes co-encapsulating doxorubicin and curcumin, through the use of a quality-by-design approach, Drug Des. Devel. Ther. 11 (2017) 1605-1621; https:// doi.org/10.2147/DDDT.S129008

26. B. Kovács, L. K. Kántor, M. D. Croitoru, É. K. Kelemen, M. Obreja, E. E. Nagy, B. Székely-Szentmiklósi and Á. Gyéresi, Reversed phase HPLC for strontium ranelate: Method development and validation applying experimental design, Acta Pharm. 68 (2018) 171-183; https://doi.org/10.2478/ acph-2018-0019

27. P. Shah, T. Pandya, M. Gohel and V. Thakkar, Development and validation of HPLC method for simultaneous estimation of rifampicin and ofloxacin using experimental design, J. Taibah Univ. Sci. 13 (2018) 146-154; https://doi.org/10.1080/16583655.2018.1548748

28. User guide to MODDE Version 12, Sartorius Stedim Data Analytics, Umeå, Sweden, 2017; https:// blog.umetrics.com/hubfs/Download\%20Files/MODDE\%2012.0.1\%20User\%20Guide.pdf; last access date November 12, 2019

29. U.S. Food and Drug Administration, Center for Drug Evaluation and Research, Dissolution Methods, FDA, Silver Spring (MD), USA, 2012; https:/www.accessdata.fda.gov/scripts/cder/dissolution/ dsp_SearchResults.cfm, last access date November 14, 2019 